\title{
Liquid level sensing by use of digital formatted optical spectrum spreading technique
}

W. Zhang

w.zhang@aston.ac.uk

K. Sugden

\section{S. Grice}

\section{Bennion}

Photonics Research Group, Aston University, Birmingham, B4 7ET, UK.

Photonics Research Group, Aston University, Birmingham, B4 7ET, UK.

Photonics Research Group, Aston University, Birmingham, B4 7ET, UK.

Photonics Research Group, Aston University, Birmingham, B4 7ET, UK.

We propose a novel technique for optical liquid level sensing. The technique takes advantage of an optical spectrum spreading technique and directly measures liquid level with a digital format. The performance of the sensor does not suffer from changes of environmental variables and system variables. Due to its distinct measurement principle both high resolution and a large measurement range can be achieved simultaneously. [DOI: 10.2971/jeos.2009.09022]

Keywords: liquid level sensing, spectrum spreading, phase grating

\section{INTRODUCTION}

There has been consistent interest in executing continuous liquid-level measurements in numerous areas, such as the fuel, oil, and water tanks of aircraft, rockets, and automobiles. In many cases, electrical sensors are inappropriate because of the hazards posed by sparks in potentially explosive environments. Electrical sensors are also be undesirable when electromagnetic interference can overwhelm the signals generated by them. Liquid level sensors using optical fibres hold particular promise as replacements for many existing sensing technologies, especially in harsh environments. Sensors based on purely optical techniques have been described (e.g. [1]-[4]). However, the performances of the techniques based on the measurements of strain, pressure and weight are greatly affected by environmental variables such as temperature, pressure, gravity, density, absorption and refractive index, also by system variables such as source intensity, attenuation. High resolution monitoring of liquid level was reported using light reflected from the liquid surface to perform an optical radar measurement of the liquid surface proximity [5]. This radar sensor is extremely sensitive to extraneous effects such as surface ripples and tilt, however, and requires a rather sensitive phase measurement.

In this paper, we describe a novel technique for optical liquid level sensing. The technique takes advantage of optical spectrum spreading technique and directly measures liquid level with a digital format. Therefore the performance of the sensor does not suffer the changes of environmental variables and system variables. The measurement resolution demonstrated in the experiments is $1 \mathrm{~mm}$. Due to its distinct operating principle both high resolutions and large measurement range can be achieved without compromising each other.

\section{PROPOSED SCHEME FOR LIQUID LEVEL SENSING}

The basic configuration of a liquid level sensing system employing the proposed technique is shown in Figure 1. It is based on optical spectrum spreading technique. The light from the broadband source (BBS) is coupled through a $3 \mathrm{~dB}$ fibre coupler and exits a GRIN lens and launched onto a dispersive prism. Owing to the dispersive effect of the prism the light at different wavelengths will be diffracted in different angles. An objective lens is used so that the light through the lens is perpendicularly incident on the mirror. Therefore the light from the broadband source is spread in $x$-direction and projected as a straight line on the mirror and reflected back along the same path. A metal tape that has encoded slots is positioned between the lens and the mirror so the reflected

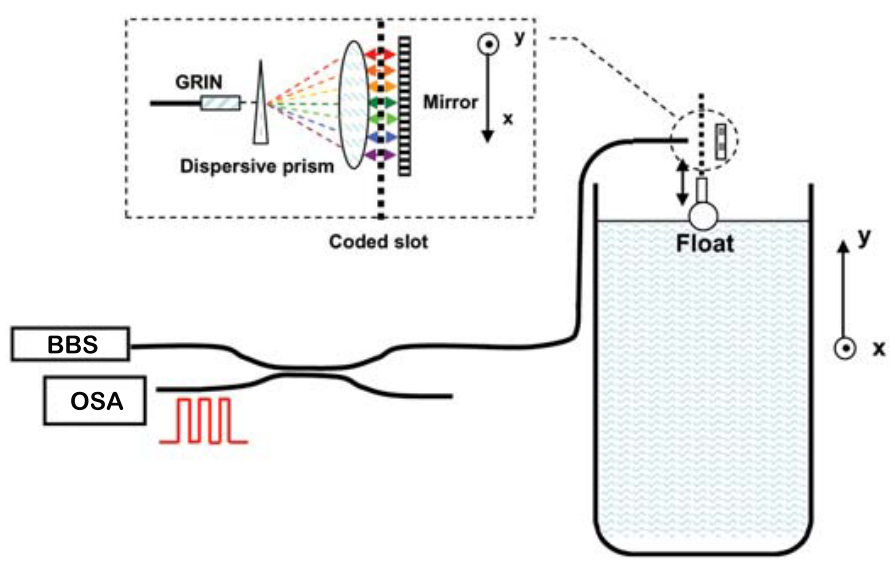

FIC. 1 Schematic diagram of proposed liquid level sensing system. 
light signal will be encoded by the pattern of the slots. The slots can be simply binary coded or gray coded in $x$-direction and moved by a float in $y$-direction. Therefore the light signal will be modulated by the slots, showing some on-off pattern along $x$-direction. The light signal at some wavelengths will be blocked and some at other wavelengths will pass through and then be reflected back through the tape to be recollected by the GRIN lens on the way back into the fibre. The reflected light is then interrogated - in the experiment by an optical spectrum analyzer (OSA). Therefore the received light signal by the OSA will be encoded in wavelength domain and each encoded spectrum represents a position in $y$-direction. Since the slot is attached to the float any liquid level change will move the slot in $y$-direction thus generating a specifically encoded light signal, as demonstrated in Figure 2. By looking at the received light signal one can precisely determine the corresponding liquid level.

\section{EXPERIMENT}

An experimental arrangement was established to verify the proposed technique, as shown in Figure 3(a). The light from a broadband source was launched into a $3 \mathrm{~dB}$ fibre coupler and exited the fibre through a GRIN lens in a collimated form, and projected onto a diffraction phase grating. The phase grating, whose first diffraction order is the maximum, enables the collimated light with different wavelength to be diffracted in different angles. The light spot on the diffraction grating is positioned at the focus point of the objective lens so that the light through the lens is perpendicularly incident on the mirror as a straight line and reflected back along the same path. The length $(L)$ of the formed straight line on the mirror, which also is the length of the encoded slot covered by the light beam, is demonstrated as

$$
L=\frac{f \cdot \Delta \lambda}{d}
$$

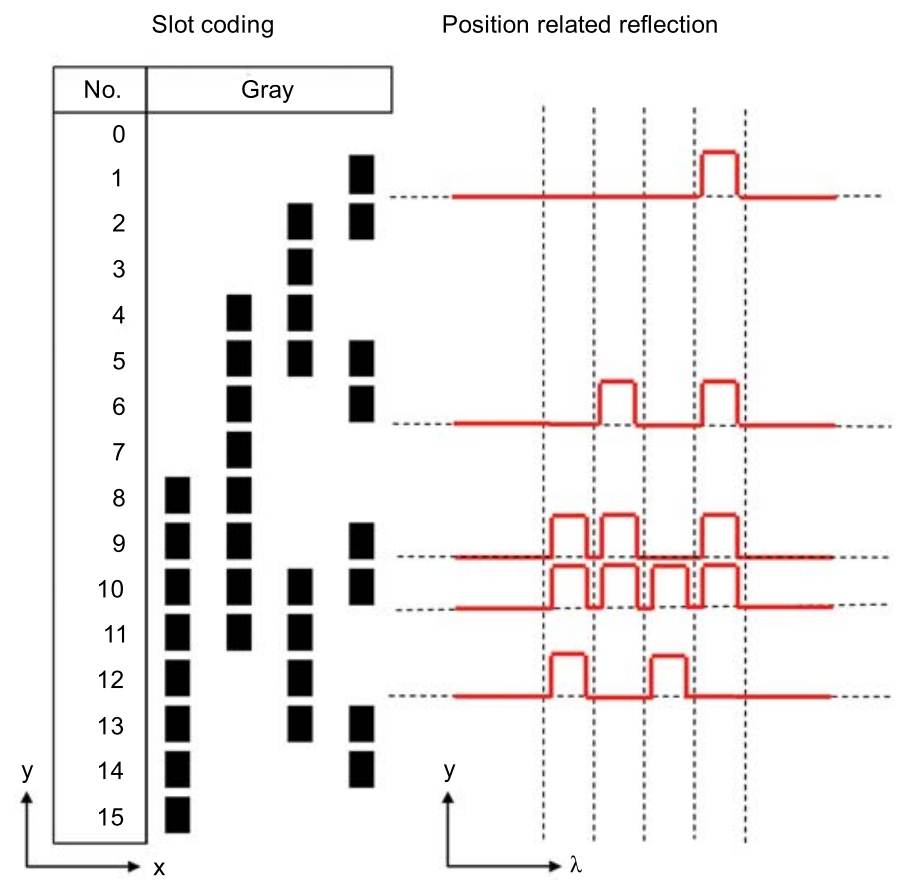

FIG. 2 Gray encoded slots and corresponding optical reflection at receiver. (a)

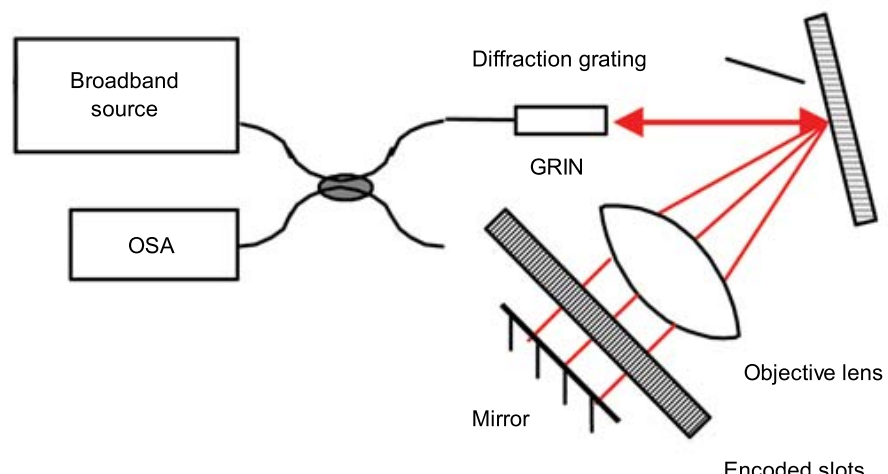

(b)

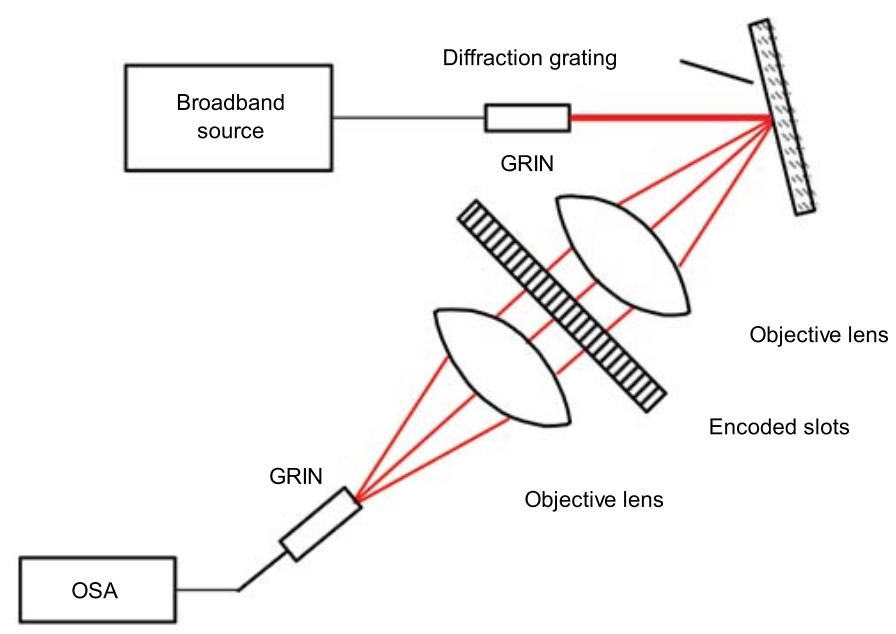

FIG. 3 Proposed sensor arrangement (a) reflection mode, (b) transmission mode.

where $f$ is the focal length of objective lens, $d$ is the pitch of phase grating and $\Delta \lambda$ is the wavelength range of the broadband source. The reflected signals are recorded by an optical spectrum analyser (OSA).

An alternative structure of the sensing system can be arranged as shown in Figure 3(b), where the optical signal operates in transmission mode rather reflected by a mirror. Instead, after passing through the encoded slots the collimated light beam can be re-focused by a second objective lens and collected by a GRIN lens into a receiving fibre, and monitored by the OSA. This structure requires an additional objective lens and GRIN lens. However, it can ease the alignment of the optics. In the experiment we chose the reflection mode as it takes less space than the transmission mode does.

The alignment of the optical circuit was carried out with the help of a tunable laser and an infrared viewing card. After the completion of the alignment the light beam produced a linear spatial scanning of $\sim 5 \mathrm{~mm}$ on the mirror while the tuneable laser was tuned from $1530 \mathrm{~nm}$ to $1580 \mathrm{~nm}$. The reflected signal from the mirror shows a flat response over $50 \mathrm{~nm}$ wavelength range as shown in the Figure 4 while the encoded slots is not placed.

The reflected light signal can be digitally coded from binary- 


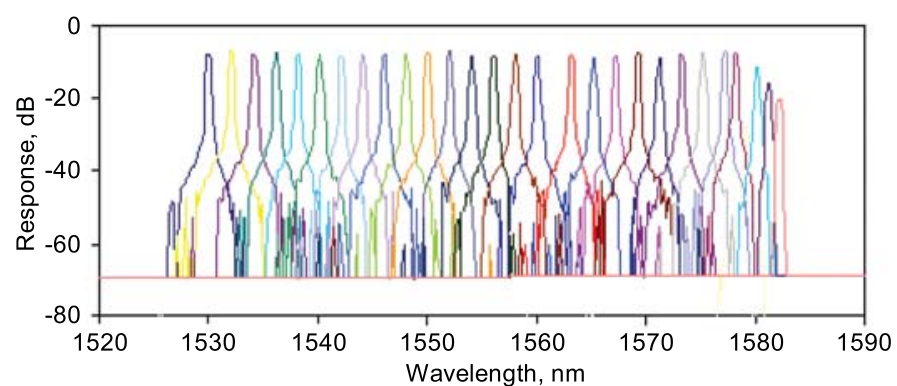

FIG. 4 Spectral response of sensing system without encoded slots.

coded mirror by using encoded slots. As illustrated in Figure 2 a metal strip with 4-digit gray encoded slots was designed and mounted on a translation stage to simulate the liquid level controlled slots. In the experiment the light spot projected on the mirror was estimated having a size of $3 \mathrm{~mm}$ (length in $x$ direction) by $0.5 \mathrm{~mm}$ (height in $y$-direction). The length of the spot corresponds to a wavelength range of $30 \mathrm{~nm}$. By tuning the translation stage so to move the coded slots in $y$ direction

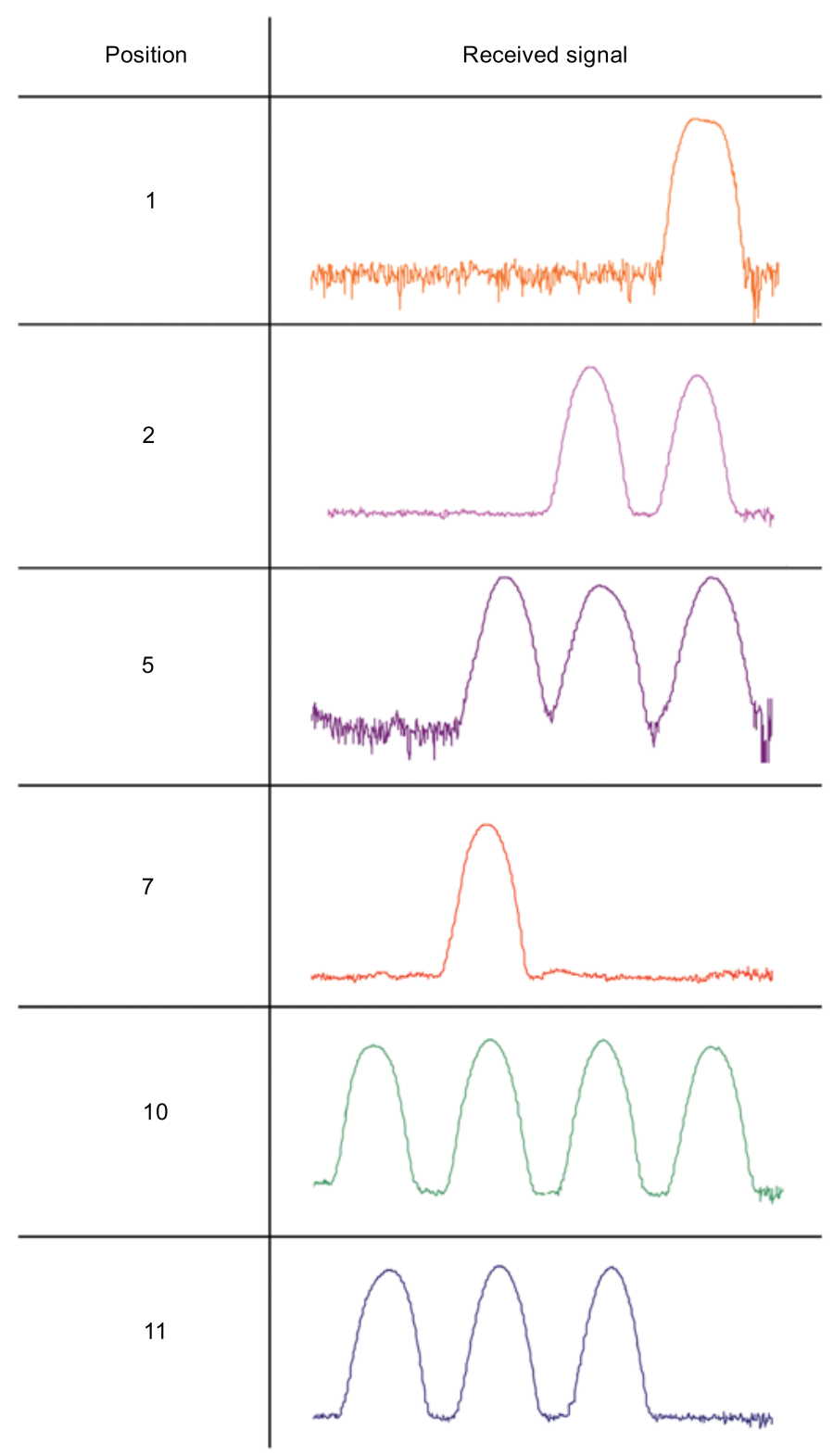

FIG. 5 Recorded optical spectra showing position related pattern. the reflected light signal was captured and recorded by OSA. Some typical recorded spectra are shown in Figure 5.

\section{RESULT AND DISCUSS}

From the recorded spectra it can be seen that each position has a distinct coded pattern thus each captured spectrum corresponds to a distinct position without ambiguity. For the 4digit coded slots 16 positions can be distinguished.

The distance between the adjacent two positions represents a minimum detectable level (resolution), which is limited by the height of the light spot. In the experiment this resolution was set as $1 \mathrm{~mm}$ due to the spot height of $0.5 \mathrm{~mm}$.

Obviously the measurement range is determined by the code length. The measurement resolution may be kept constant as long as the height of the spot is the same. If the code length increases to 8 digits the measured position can be up to 256, equal to $256 \mathrm{~mm}$ of liquid level with a resolution of $1 \mathrm{~mm}$. For a spot length of $3 \mathrm{~mm}$ it is not difficult to make a code length of 8 digits. The longer code length can be realised by employing the smaller slot size. It should be pointed out that for a very small slot size, the diffraction from the slot edges may cause the captured pattern distortion, which is a kind of bit error in this system.

There are several ways to increase code length. According to Eq. (1), increasing the focus length of the lens used generates a larger length of the light spot. Employing phase grating with smaller period or taking up larger bandwidth of the light source will equivalently give rise to larger length of the light spot on the mirror, thus accommodating more bits of slot.

The measurement resolution can be improved by reducing the spot size in $y$-direction. This can be realized by introducing a cylindrical lens between the encoded slots and the objective lens to suppress the spot in $y$-direction.

The most significant feature for this proposed technique is its digital format of the light signal representing liquid level. As one can see from the results in Figure 5 each captured trace of the reflected signal has a shape analogous to a Gray code pattern. Therefore at the receiving end, instead of an OSA one can use a combination of a second diffraction phase grating and a linear CCD array to receive the reflected light signal and generate a binary output as shown in the Figure 6. This is more pragmatic and cost effective approach for a real application. The final output is the readout of an on-off signal at the corresponding position which would definitely ease the electronic design. Due to this digital format of the light signal the sensor will not be affected by system variables such as source intensity drift, temperature, aging and changing losses due to fiber bends and connectors. Also the sensor will not be affected by fluid variables such as temperature, density, refractive index and absorption. The performance of the sensor greatly depends on the optical probe that operates as wavelength coding/decoding and can be designed in a compact and rigid form. Also the optical probe is not in direct contact with the liquid to be measured, it can be well maintained to 


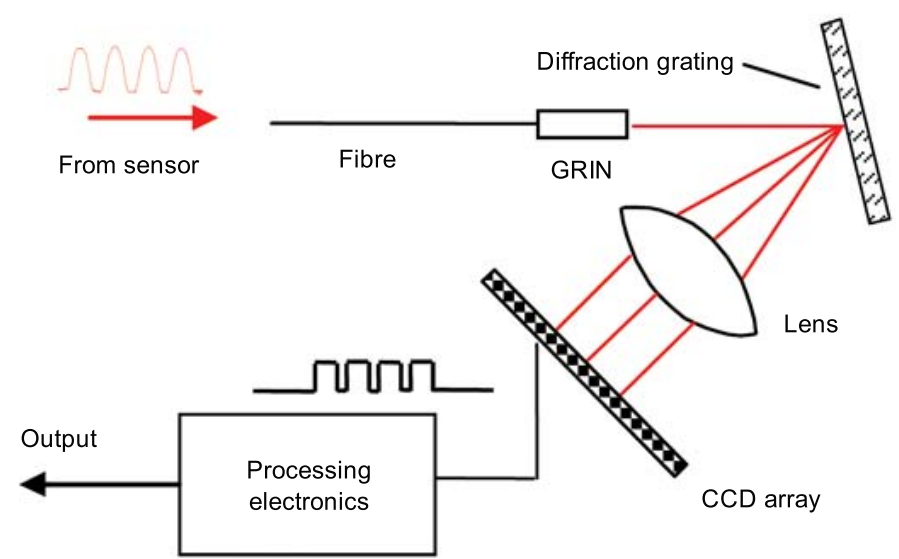

FIG. 6 Schematic for recovering sensor information.

deliver long term reliability. The sensor will not be affected by the liquid surface ripple and tilt caused by vibration, however, it is not suitable for airborne application due to the use of the moving part (float).

\section{CONCLUSION}

We have demonstrated a novel technique for the optical measurement of liquid level. The optical technique used allows the sensor to operate in a hazardous environment. The digital format of the light signal carrying the sensing information has a great advantage of being not affected by environmental and system perturbations for in situ application. The sensing principle proposed offers both large measurement range and high resolution. The technique can also be used in the applications of position or displacement measurement.

\section{ACKNOWLEDGEMENT}

The work is sponsored under the UK TSB project "Integrated Wing".

\section{References}

[1] S. F. Knowles, B. E. Jones, S. Purdy, and C. M. France, "Multiple microbending optical-fiber sensors for measurement of fuel quantity in aircraft fuel tanks" Sensor Actuat. A-Phys. 68, 330-323 (1998).

[2] S. Khaliq, S. W. James, and R. P. Tatam, "Fiber-optic liquid-level sensor using a long-period grating" Opt. Lett. 26, 1224-1226 (2001).

[3] I. P. Dakin and M. G. Holliday, A liquid level sensor based on $0-H$ or C-H absorption monitoring, 91-95 (Proc. First Int. IEE Con. Optical Fiber Sensors 221, London, England, 1983).

[4] K. E. Romo-Medrano and S. N. Khotiaintsev, "An optical-fiber refractometric liquid-level sensor for liquid nitrogen" Meas. Sci. Technol. 17, 998-1004 (2006).

[5] D. A. Jackson, High precision remote liquid level measurement using a combination of optical radar and optical fibers, 100-103 (Proc. First Int. IEE Con. Optical Fiber Sensors 221, London, England, 1983). 